\title{
SÍNTESE E CARACTERIZAÇÃO DE NOVOS COMPLEXOS DE PLATINA (II) COM LIGANTES DERIVADOS DO FURANO E NITROFURANO
}

Wendell Guerra, Ana Paula Soares Fontes*, Mauro Vieira de Almeida e Heveline Silva

Departamento de Química, Universidade Federal de Juiz de Fora, Campus Martelos, 36036-330 Juiz de Fora - MG

Recebido em 15/7/04; aceito em 24/2/05; publicado na web em 30/6/05

\begin{abstract}
SYNTHESIS AND CHARACTERIZATION OF NEW PLATINUM (II) COMPLEXES CONTAINING FURAN AND NITROFURAN DERIVED LIGANDS. Platinum (II) complexes, for example, cisplatin and carboplatin, have been used as chemotherapeutic agents for the treatment of various types of cancer. Several other complexes of this metallic ion are also under clinical evaluation. This work describes the synthesis of five new platinum (II) complexes having furan and 5-nitrofuran derivatives and chloride as ligands. The compounds were characterized by NMR, IR and elemental analysis.
\end{abstract}

Keywords: platinum (II) complexes; furan; nitrofuran.

\section{INTRODUÇÃO}

A quimioterapia do câncer utiliza-se tanto de compostos orgânicos (como por ex., taxol) quanto de compostos inorgânicos (por ex., cisplatina e carboplatina, Figura 1), que podem ser administrados sozinhos ou em associação com outras drogas.
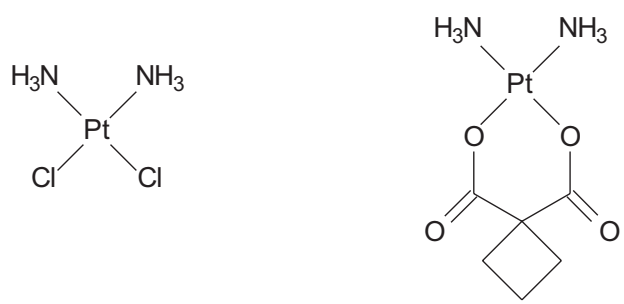

Figura 1. cisplatina e carboplatina

Há algumas décadas, a cisplatina, cis-diaminodicloroplatina(II), vem sendo usada no tratamento de vários tipos de neoplasias, como de pulmão, cabeça, esôfago, estômago, linfomas, melanoma, osteossarcoma, de mama e cérvix ${ }^{1}$. Por ex., em combinação com bleomicina e vimblastina, a cisplatina promove a cura em $80 \%$ dos casos de câncer testicular e, em combinação com paclitaxel, prolonga o tempo de vida de pacientes com câncer ovariano em estágio avançado ${ }^{2}$.

A utilização da cisplatina, entretanto, apresenta alguns pontos negativos, como aparecimento de resistência celular, pouca solubilidade em água e efeitos colaterais graves, como nefrotoxicidade, neurotoxicidade, ototoxicidade e toxicidade gastrointestinal ${ }^{3,4}$.

Acredita-se que muitos dos efeitos adversos observados, principalmente nefrotoxicidade, estejam relacionados com a ocorrência de ligação do complexo de platina com proteínas e peptídeos, como a glutationa, e a conseqüiente acumulação destes adutos no organismo $^{5}$. Sabe-se também que o mecanismo de ação de compostos de platina, em geral, envolve a interação destes com o DNA, configurando-se uma lesão em nível molecular ${ }^{6,7}$.

A preparação de novos complexos de platina tem sido direcionada para o desenvolvimento de compostos mais eficazes e menos tóxicos. Desta maneira, cerca de 3000 complexos já foram sinteti-

*e-mail: ana.fontes@ufjf.edu.br zados e testados, mas somente três outros são usados hoje: a carboplatina, a oxaloplatina e a nedaplatina ${ }^{2}$.

As pesquisas nesta área continuam, não só no sentido de se obter um análogo que apresente menos efeitos colaterais, mas que também exiba um espectro de atividade maior, principalmente no que diz respeito às células resistentes à cisplatina.

Neste trabalho discute-se a síntese e caracterização de novos complexos de platina(II) com ligantes derivados do furano e do 5 nitrofurano que representam diferentes classes de compostos químicos, como hidrazonas, hidrazidas, oximas, semicarbazonas e aminas. Nosso grupo de pesquisa publicou recentemente trabalho envolvendo a preparação de complexos de cobre (II) com dois destes derivados ${ }^{8}$. Estes ligantes apresentam atividade biológica ou pertencem à uma classe de compostos que possui atividade biológica.

Os primeiros compostos nitro-heterocíclicos a serem introduzidos na quimioterapia foram os nitrofuranos ${ }^{9}$. Em geral, os nitrofuranos apresentam atividade antibacteriana, que se deve à redução enzimática do grupo nitro in vivo, produzindo espécies tóxicas que, subseqüentemente, causam danos ao DNA das bactérias ${ }^{10}$. O grupo nitro está presente ainda em várias substâncias usadas como antitumorais, tripanocidas, fungicidas, antivirais e antiparasitários ${ }^{10,11}$. Hidrazonas, em geral, possuem diversas atividades biológicas, como anti-inflamatória, antitrombótica e analgésica ${ }^{12,13}$. Compostos do tipo nitrofurfural hidrazona têm apresentado atividade antiparasitária e antimicrobiana in vivo ${ }^{13}$. Está bem documentado que algumas oximas e seus complexos exibem atividade antiviral e citotóxica ${ }^{14}$. As hidrazidas e seus complexos podem atuar como agentes fungicidas e antibacterianos ${ }^{15}$. As semicarbazonas apresentam diversas atividades biológicas, sendo que seus complexos metálicos exibem atividade citotóxica e antiviral ${ }^{16}$.

\section{RESULTADOS E DISCUSSÃO}

\section{Síntese e caracterização dos ligantes}

Dentre os ligantes utilizados, mostrados na Figura 2, dois foram sintetizados e caracterizados em nossos laboratórios (a e b) e três foram obtidos comercialmente (c, d e e).

Para a obtenção do composto b, foi feita a reação do 2furanocarboxaldeído com cloridrato de semicarbazida. Já para a 


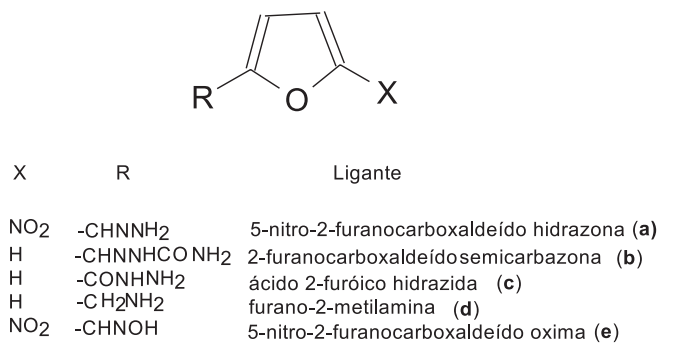

Figura 2. Ligantes utilizados

obtenção do derivado do 5-nitrofurano, composto a, utilizou-se como reagente de partida o diacetato de 5-nitro-2-furanocarboxaldeído. Na Figura 3 apresenta-se um esquema de síntese dos ligantes a e b preparados em uma única etapa.

Devido à possibilidade de formação de isômeros E e Z, utilizou-se banho de gelo para favorecer a formação de um único isômero. Não foi necessário purificar os ligantes sintetizados por coluna cromatográfica. Após a obtenção e filtração, foi feita apenas uma lavagem do sólido obtido com um solvente apropriado. Os rendimentos obtidos nas reações de síntese destes ligantes foram satisfatórios.

Os ligantes sintetizados foram caracterizados por ponto de fusão, espectroscopia na região do infravermelho, RMN de ${ }^{1} \mathrm{H}, \mathrm{RMN}$ de ${ }^{13} \mathrm{C}$ e por análise elementar, enquanto para os comerciais foram realizados espectros na região do infravermelho, RMN de ${ }^{1} \mathrm{H}$ e RMN de ${ }^{13} \mathrm{C}$ com o objetivo de compará-los com os espectros dos complexos.

Os espectros de infravermelho dos ligantes $\mathbf{a}, \mathbf{b}, \mathbf{c}$ e $\mathbf{d}$ apresentaram absorções características nas regiões de 3469 a $3147 \mathrm{~cm}^{-1}$ correspondentes a $v_{\mathrm{NH}}$. Nos espectros de todos os ligantes são observadas absorções correspondentes a $v_{\mathrm{CH} \text { (furânico) }}$ na região de 3100 a $3021 \mathrm{~cm}^{-1}$. Com exceção do ligante c, observam-se também as absorções relativas a $v_{\mathrm{CH}(\text { alifático) }}$ em frequiências um pouco menores. Além destas absorções, os espectros dos ligantes a, b e e mostraram $v_{\mathrm{C}=\mathrm{N}}$ na região de 1655 a $1584 \mathrm{~cm}^{-1}$ e os espectros dos compostos b e c apresentaram $v_{\mathrm{C}=\mathrm{O}}$ em 1695 e $1657 \mathrm{~cm}^{-1}$, respectivamente. Para o ligante e foi possível observar ainda uma banda em $3449 \mathrm{~cm}^{-1}$, correspondente a $\mathrm{v}_{\mathrm{OH}}$. Nos espectros deste último ligante e também no do ligante a, ocorrem ainda absorções relativas ao grupo nitro em torno de 1550 e $1340 \mathrm{~cm}^{-1}$.

Nos espectros de RMN de ${ }^{1} \mathrm{H}$, os sinais correspondentes aos hidrogênios do anel furânico dos ligantes a e e ocorreram como dois dupletos na região de $\delta 6,72$ a 7,72. Para os outros três ligantes, como era esperado, observaram-se três sinais, que apresentaram maior desdobramento, na região de $\delta 6,60$ a 7,79 relativos aos hidrogênios do anel aromático. Os sinais de ressonância relativos ao hidrogênio do carbono alifático dos ligantes a, b e e ocorreram como simpletos em $\delta 7,55 ; 7,74$ e 7,76, respectivamente. Os sinais de hidrogênio ligados aos átomos de nitrogênio foram observados como simpletos largos em regiões variadas. Finalmente, para o ligante e observou-se um sinal adicional em $\delta 12,64$, correspondente ao hidrogênio da hidroxila.

Foram realizados ainda espectros de $\mathrm{RMN}$ de ${ }^{13} \mathrm{C}$ para os ligantes a, c e e. Para os compostos a e e, os sinais correspondentes ao carbono aromático ligado ao grupo nitro ocorreram em $\delta 155$ e 150 , respectivamente. Os sinais dos carbonos aromáticos ligados a hidrogênio ocorreram em $\delta 108$ e 116 para a e $\delta 114$ e 118 para e. Ainda para estes dois compostos, os sinais do quarto carbono aromático foram observados em $\delta 150$ e 146, respectivamente, para os ligantes a e e. Os sinais de ressonância relativos ao carbono alifático de a e e, ocorreram em $\delta 123$ e 134, respectivamente. Para o ligante c, observaram-se os sinais de ressonância dos carbonos aromáticos em $\delta 144$ e 147 (carbonos vizinhos ao heteroátomo) e $\delta 111$ e 112. O sinal de ressonância correspondente ao carbono alifático ocorreu em $\delta 152$.

\section{Síntese e caracterização dos complexos}

A partir dos ligantes apresentados, foram sintetizados cinco novos complexos de platina (II) (complexos 1, 2, 3, 4 e 5, Figura 4).

Os complexos foram obtidos a partir da reação de $1 \mathrm{mmol}$ de $\mathrm{K}_{2} \mathrm{PtCl}_{4}$ com 2 mmols do ligante apropriado. As condições das reações, como solvente utilizado, temperatura e tempo, variaram conforme descrito na parte experimental. Todos os complexos obtidos foram separados por simples filtração e são solúveis em dimetilformamida e dimetilssulfóxido.

Os compostos sintetizados foram caracterizados por espectroscopia na região do infravermelho, RMN de ${ }^{195} \mathrm{Pt}$, RMN de ${ }^{1} \mathrm{H}, \mathrm{RMN}$ de ${ }^{13} \mathrm{C}$ e por análise elementar.

Nos espectros de infravermelho dos complexos foi possível observar uma banda na freqüência de 318 a $332 \mathrm{~cm}^{-1}$, dependendo do complexo, característica de $\mathrm{v}_{\mathrm{Pt}-\mathrm{Cl}}$. Esta banda apresentou-se desdobrada, o que é característico da geometria cis para complexos quadradros planares de platina (II) ${ }^{17}$. Foram observadas ainda as bandas de absorção dos ligantes. Em comparação com os espectros dos ligantes livres, observou-se que a banda relativa a $v_{\mathrm{NH}}$ deslocou-se para menor freqüência nos complexos 1 a 4 e apresentou-se com melhor definição devido à menor possibilidade de formação de ligação de hidrogênio, uma vez que o átomo de nitrogênio está envolvido na coordenação com a platina ${ }^{18}$. Para o complexo 5, observou-se ainda um deslocamento para menor frequiência da banda característica de $\mathrm{v}_{\mathrm{C}=\mathrm{N}}$, coerente com o envolvimento deste átomo de nitrogênio na coordenação com a platina.

Os espectros de RMN de ${ }^{1} \mathrm{H}$ e RMN de ${ }^{13} \mathrm{C}$ para os complexos foram realizados. Entretanto, não apresentaram informações adicionais úteis para a caracterização dos mesmos, estando, porém,

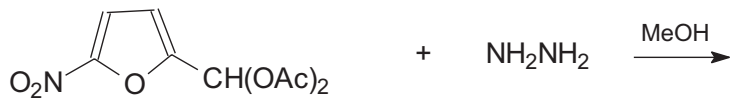

diacetato de 5-nitro-2-furanocarboxaldeído<smiles>O=Cc1ccco1</smiles>

$+\mathrm{NH}_{2} \mathrm{NHCONH} \mathrm{H}_{2} \mathrm{HCl}$

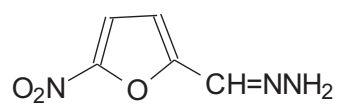

ligante a 
<smiles>[NH3+][P+](Cl)(N=Cc1ccc([N+](=O)[O-])o1)N=Cc1ccc([N+](=O)[O-])o1</smiles>

complexo 1

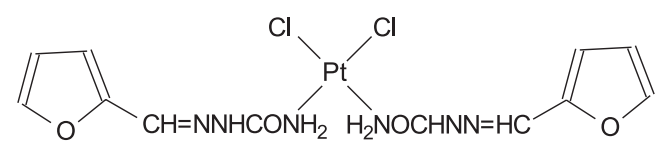

complexo 2<smiles>NNP(Cl)(Cl)(NN)NOC(=O)c1ccco1</smiles>

complexo 3<smiles>NP(N)(Cl)(Cl)Cc1ccco1</smiles>

complexo 4<smiles></smiles>

complexo 5

Figura 4. Complexos sintetizados

coerentes com as estruturas propostas. Estes espectros foram muitas vezes realizados em dimetilssulfóxido (DMSO) deuterado e, em alguns casos, observou-se o deslocamento parcial do ligante pelo solvente. A solvólise de complexos de platina em DMSO é bastante comum e está bem documentada na literatura ${ }^{19,20}$. Em RMN de ${ }^{1} \mathrm{H}$, a ligação do DMSO à platina é evidenciada pelo aparecimento de um sinal de ressonância por volta de $\delta 3,5$, correspondente aos hidrogênios metílicos do DMSO- $d_{\sigma}$, que no solvente livre ocorre por volta de $2,5^{21}$.

Todos os complexos apresentaram um único sinal de ressonância em RMN de ${ }^{195} \mathrm{Pt}$. Considerando-se a importância desta técnica para a caracterização dos complexos, em alguns casos os espectros foram feitos em dimetilformamida (DMF) para evitar a solvólise observada com DMSO. Os complexos apresentaram sinal entre $\delta-2321$ e -2226 . Este valor de deslocamento químico está condizente com complexos similares encontrados na literatura, nos quais a platina apresenta na sua esfera de coordenação dois cloretos e dois átomos de nitrogênio ${ }^{22}$.

\section{PARTE EXPERIMENTAL}

Os ligantes c (ácido 2-furóico hidrazida), d (furano-2metilamina) e e (5-nitro-2-furanocarboxaldeído oxima) foram obtidos da Aldrich e utilizados sem purificação prévia. Os demais reagentes e solventes tinham grau de pureza P. A. e foram adquiridos da Aldrich, Fluka, Vetec e Merk, tendo sido usados também sem prévia purificação.
Os espectros na região do infravermelho foram feitos no DQ da UFJF num espectrofotômetro Bomem FT IR MB-102, na região de 4000 a $300 \mathrm{~cm}^{-1}$, utilizando como suporte pastilhas de $\mathrm{KBr}$.

As análises elementares de carbono, hidrogênio e nitrogênio dos compostos obtidos neste trabalho foram feitas na Central Analítica do IQ da USP.

Os espectros de RMN de ${ }^{1} \mathrm{H}$, de ${ }^{13} \mathrm{C}$ e de ${ }^{195} \mathrm{Pt}$ foram feitos no LAREMAR da UFMG utilizando um espectrômetro Brucker Avance DRX 200 e DRX 400. Os deslocamentos químicos foram expressos em $\delta$ a partir do padrão interno TMS (RMN de ${ }^{1} \mathrm{H}$ ) e de uma solução de $\mathrm{K}_{2} \mathrm{PtCl}_{4}$ em $\mathrm{D}_{2} \mathrm{O}\left(\mathrm{RMN}\right.$ de $\left.{ }^{195} \mathrm{Pt}\right)$.

\section{Síntese dos ligantes}

5-nitro-2-furanocarboxaldeído hidrazona (a)

A uma solução de diacetato de 5-nitro-2-furanocarboxaldeído $(0,486 \mathrm{~g} ; 2 \mathrm{mmol})$ em metanol $(20 \mathrm{~mL})$ resfriada em banho de gelo, adicionou-se, gota-a-gota, hidrato de hidrazina a $65 \%(0,3$ $\mathrm{mL}$ ). Após permanecer em agitação por $18 \mathrm{~h}$, o consumo dos reagentes de partida foi evidenciado por CCDS (eluente hexano/ acetato 8:2). O sólido laranja obtido foi filtrado e lavado com água destilada, metanol e etanol. Depois de seco, obteve-se 0,206 g de produto, correspondendo a um rendimento de $67 \%$.

IV. $v \mathrm{KBr}\left(\mathrm{cm}^{-1}\right): 3441,3168,3090,3021,1584,1551,1505$, 1474, 1384, 1342, 1239, 1232(..); RMN de ${ }^{1} \mathrm{H}$ (200 MHz; DMSO$\left.d_{6}\right) \delta 7,96 ; 7,55\left(2 \mathrm{~s}, 1 \mathrm{NH}_{2}, 1 \mathrm{CH}\right.$ alifático $) ; 7,65 ; 6,72(2 \mathrm{~d}, 2 \mathrm{H}$ furânicos, $\mathrm{J}=3,97 \mathrm{~Hz})$; RMN de ${ }^{13} \mathrm{C}\left(50 \mathrm{MHz}\right.$; DMSO- $\left.d_{6}\right) \delta 108$, 116 (C do anel furânico não vizinho ao oxigênio), 123 (C alifático), 150 (C do anel furânico ligado ao grupo R), 155 (C do anel furânico ligado ao grupo nitro); análise elementar calculada para $\mathrm{C}_{5} \mathrm{H}_{5} \mathrm{O}_{3} \mathrm{~N}_{3}$ : C 38,71; H 3,23; N 27,10; encontrada: C 39,29; H 3,43; N 26,31.

\section{2-furanocarboxaldeído semicarbazona (b)}

A uma solução de cloridrato de semicarbazida (1 g) e acetato de sódio (1,5 g) em água e metanol, resfriada em banho de gelo, adicionou-se, sob agitação, 2-furanocarboxaldeído (0,5 mL, $6 \mathrm{mmol})$. Imediatamente observou-se a formação de um sólido amarelado, que foi filtrado e lavado com éter etílico. Depois de seco, obteve-se 0,77 $\mathrm{g}$ de produto, correspondendo a um rendimento de $82 \%$.

IV. $\vee \mathrm{KBr}\left(\mathrm{cm}^{-1}\right): 3450,3290,3100,2998,1695,1655$ (...); RMN de ${ }^{1} \mathrm{H}\left(200 \mathrm{MHz}\right.$; acetona- $\left.d_{6}\right) \delta 10,23 ; 7,74$ (2s, H do grupo $\mathrm{NH}$ e $\mathrm{H}$ do grupo $\mathrm{CH}$ alifático); 6,31 ( $\mathrm{H}$ do grupo $\left.\mathrm{NH}_{2}\right) ; 7,73$ (H do carbono vizinho ao oxigênio do anel furânico); 6,70; 6,60 (d e dd dos $\mathrm{H}$ furânicos); análise elementar calculada para $\mathrm{C}_{6} \mathrm{H}_{7} \mathrm{O}_{2} \mathrm{~N}_{3}: \mathrm{C}$ 47,06; H 4,58; N 27,45; encontrada: C 48,13; H 4,40; N 26,76.

\section{Síntese dos complexos}

A uma solução de $\mathrm{K}_{2} \mathrm{PtCl}_{4}(0,208 \mathrm{~g} ; 0,5 \mathrm{mmol})$ em água (5 mL) foi adicionado o ligante apropriado (1 mmol) em solução de metanol (20 mL, ligante a), suspensão em água (ligante b) ou solução em água ( $5 \mathrm{~mL}$, ligantes $\mathbf{c}, \mathbf{d}$ e e). Após agitação por um período de 20 a 70 h, dependendo do complexo, à temperatura ambiente, evidenciou-se a formação de um precipitado e o consumo do ligante por CCDS (eluente: acetato de etila/hexano 1:1). O produto foi isolado por filtração, lavado com água e metanol e seco. Rendimentos: 1 $(0,210 \mathrm{~g} ; 73 \%) ; 2$ (0,234 g; 82\%); 3 (0,466 g; 90\%); 4 (0,125 g; $54 \%) ; 5(0,18 \mathrm{~g} ; 62 \%)$.

1: IV $\vee \mathrm{KBr}\left(\mathrm{cm}^{-1}\right): 3400,3150,3128,3097,1638,1564,1514$, $1472,1396,1351,325,318 ; \mathrm{RMN}$ de ${ }^{1} \mathrm{H}\left(200 \mathrm{MHz}\right.$; DMF- $\left.d_{7}\right): \delta$ 8,7 e 8,0 (2 s, CH alifático e $\left.\mathrm{NH}_{2}\right) ; \delta 7,8 ; 7,5(2 \mathrm{~d}, \mathrm{~J}=3,91 \mathrm{~Hz}$, hidrogênios furânicos); RMN de ${ }^{195} \mathrm{Pt}\left(86 \mathrm{MHz}\right.$; DMF- $\left.d_{7}\right) \delta-2321$; análise elementar calculada para $\mathrm{C}_{10} \mathrm{H}_{10} \mathrm{O}_{6} \mathrm{~N}_{6} \mathrm{PtCl}_{2}$ : C 20,83; $\mathrm{H} 1,74$; 
N 14,58; encontrada: C 21,83; H 1,87; N 13,71.

2: IV $\vee \mathrm{KBr}\left(\mathrm{cm}^{-1}\right): 3457,3283,3151,3003,1691,1654,1602$, 1516, 324, 320; RMN de ${ }^{195} \mathrm{Pt}$ (86 MHz; DMSO- $\left.d_{6}\right) \delta$ - 2220; análise elementar calculada para $\mathrm{C}_{12} \mathrm{H}_{14} \mathrm{O}_{4} \mathrm{~N}_{6} \mathrm{PtCl}_{2}: \mathrm{C} 25,17 ; \mathrm{H} \mathrm{2,45; \textrm {N }}$ 14,69; encontrada: C 25,85; H 2,59; N 15,63.

3: IV $\vee \mathrm{KBr}\left(\mathrm{cm}^{-1}\right)$ : 3210, 3041, 1648, 1589, 1523, 1457, 1324, 329, 325; RMN de ${ }^{195} \mathrm{Pt}$ (86 MHz; DMSO- $\left.d_{6}\right) \delta$ - 2226; análise elementar calculada para $\mathrm{C}_{10} \mathrm{H}_{12} \mathrm{O}_{4} \mathrm{~N}_{4} \mathrm{PtCl}_{2}$ : C 23,17; $\mathrm{H} 2,32 ; \mathrm{N}$ 10,81; encontrada: C 23,20; H 2,24; N 10,82.

4: IV $\vee \mathrm{KBr}\left(\mathrm{cm}^{-1}\right): 3400,3224,3200,3100,1576,1503,332$, 329; RMN de ${ }^{195} \mathrm{Pt}\left(86 \mathrm{MHz}\right.$; DMSO- $d_{6}$ ) $\delta$ - 2226; análise elementar calculada para $\mathrm{C}_{10} \mathrm{H}_{14} \mathrm{O}_{2} \mathrm{~N}_{2} \mathrm{PtCl}_{2}$ : C 26,09; $\mathrm{H} \mathrm{3,04;} \mathrm{N} \mathrm{6,09;} \mathrm{en-}$ contrada: C 25,60; H 2,70; N 5,61.

5: IV $\vee \operatorname{KBr}\left(\mathrm{cm}^{-1}\right): 3160,3005,1617,1530,1477,1351,1352$, 325, 321; RMN de ${ }^{1} \mathrm{H}\left(200 \mathrm{MHz}\right.$; DMF- $\left.d_{7}\right)$ : $\delta$ 7,04 e 7,71 (2 d, hidrogênios do anel furânico); $\delta 8,15$ e 12,16 (2 s, CH alifático e hidroxila); RMN de ${ }^{195} \mathrm{Pt}\left(86 \mathrm{MHz}\right.$; $\left.\mathrm{DMF}-d_{7}\right) \delta-2239$; análise elementar calculada para $\mathrm{C}_{10} \mathrm{H}_{8} \mathrm{O}_{8} \mathrm{~N}_{4} \mathrm{PtCl}_{2}$ : C 20,76; $\mathrm{H} \mathrm{1,38;} \mathrm{N} \mathrm{9,69;}$ encontrada: C 21,79; H 1,48; N 9,73.

\section{CONCLUSÕES}

Este trabalho descreveu a síntese de novos complexos de platina (II) com ligantes derivados do furano e nitrofurano, que foram sintetizados ou obtidos comercialmente. Os ligantes e os complexos foram caracterizados por espectroscopia na região do infravermelho, RMN de ${ }^{1} \mathrm{H}$, RMN de ${ }^{13} \mathrm{C}$, RMN de ${ }^{195} \mathrm{Pt}$ e por análise elementar.

Tendo em vista o tipo de ligante utilizado e as estruturas dos complexos obtidos, estes poderão apresentar atividade biológica.

\section{AGRADECIMENTOS}

À FAPEMIG pelo apoio financeiro. W. Guerra agradece à CAPES pela bolsa concedida.

\section{REFERÊNCIAS}

1. Fiorentino, M. V.; Ghiotto, C.; Inorg. Chim. Acta 1987, 59, 13.

2. Coluccia, M.; Natile, G.; Coord. Chem. Rev. 2001, 384, 216.

3. Barnad, C. F. J.; Cleare, M. J.; Hydes, P. C.; Chemistry in Britain 1986, $22,1001$.

4. Ozols, R. F.; Seminars in Oncology 1989, 16, 22.

5. Appleton, T. G.; Connor, J. W.; Hall, J. R.; Prenzler, P. D.; Inorg. Chem. 1989, 28, 2030.

6. Lippard, S. J.; Pure Appl. Chem. 1987 59, 731.

7. Fontes, A. P.; de Almeida, S. G.; Nader, L.; Quim. Nova 1997, 20, 398.

8. Fontes, A. P. S.; Guerra, W.; Machado, F. C.; de Almeida, M. V.; Alves, W. A.; Ferreira, A. M. C.; Paduan-Filho, A.; Transition Met. Chem. 2004, 29, 382.

9. Pires, J. R.; Saito, C.; Gomes, S. L.; Giesbrecht, A. M.; T. do Amaral, A.; J. Med. Chem. 2001, 44, 3673.

10. de Almeida, M. V.; le Hyaric, M.; Siquiera, L. J. A.; Pinto, L. D.; Valle, M. S.; Alves, W. A.; Molecules 2001, 6, 728 .

11. Novinson, T.; Bhooshan, B.; Okabe, T.; Revankar, G. R.; Robins, R. K.; Senga, K.; Wilson, H. R.; J. Med. Chem. 1976, 19, 4.

12. Barreiro, E. J.; Fraga, C. A. M.; Miranda, L. P.; Rodrigues, C. R.; Quim. Nova 2002, 25, 129.

13. Ainscough, E. W.; Brodie, A. M.; Dobbs, A. J.; Ranford, J. D.; Waters, J. M.; Inorg. Chim. Acta 1998, 267, 27.

14. Ghassan, I.; Bouet, G. M.; Hall, I. H.; Khan, M. A.; J. Inorg. Biochem. 2000, 81, 29.

15. Dodoff, N.; Granharov, K.; Spassovska N.; J. Inorg. Biochem. 1995, 60, 257.

16. Fahmi, N.; Singh, R. V.; J. Indian Chem. Soc. 1996, 73, 257.

17. Nakamoto, K.; Infrared and Raman Spectra of Inorganic and Coordination Compounds, $3^{\text {rd }}$ ed., Wiley: New York, 1976.

18. César, E.T; de Almeida, M. V.; Fontes, A. P. S.; Maia, E. C. P.; GarnierSuillerot, A.; Couri, M. R. C.; Felício, E. C. A.; J. Inorg. Biochem. 2003, 95, 297.

19. Kerrison, S.; Sadler, P. J.; Inorg. Chim. Acta 1985, 104, 197.

20. Fanizzi, F. P.; Intini, F. P.; Maresca, L.; Natile, G.; Uccello-Barretta, G.; Inorg. Chem. 1990, 29, 29.

21. Farrell, N.; Kiley, D.; Schmidt, W.; Hacker, M.; Inorg. Chem. 1990, 29, 397.

22. de Almeida, M. V.; César, E.T.; Felício, E. C. A.; Fontes, A. P. S.; J. Braz. Chem. Soc. 2000, 11, 154. 